\title{
Leistungsverweigerung aus Gewissensgründen
}

Die Standesregeln sehen vor, dass der Arzt von Leistungen, die ihn in einen Gewissenskonflikt bringen, Abstand nehmen kann. Dies gilt für die Abtreibung, die Verschreibung von Verhütungsmitteln (inkl. Sterilisation) oder die medizinisch unterstützte Fortpflanzung.

Wenn ich jedoch als Kantonsarzt konsultiert wurde, erinnerte ich daran, dass die Verweigerung ärztlicher Leistungen deontologisch nur unter gewissen Voraussetzungen zulässig ist:

- Nicht zulässig wäre sie in einer Notsituation, in der das Ausbleiben einer Behandlung oder deren Verzögerung negative Konsequenzen für den Patienten hätte;

- ebenfalls nicht zulässig wäre sie für den Fall, dass der/die Betroffene ernsthafte Schwierigkeiten hätte, besagte Leistung anderweitig zu erhalten - beispielsweise aufgrund der Entfernung oder erheblicher materieller und finanzieller Konsequenzen ...;

- selbst wenn er das Vorhaben des Patienten nicht gutheissen kann, ist der Arzt deontologisch verpflichtet, ihn adäquat über jene Kollegen zu informieren, die die Leistung erbringen.

Problematisch würde es - beispielsweise im Bereich der Gynäkologie -, wenn alle Ärzte in einem relativ grossen Gebiet eine Pflegeleistung aus Gewissensgründen verweigerten. Dann änderte sich die Sachlage. Was aus Respekt vor der Meinung des einzelnen zulässig ist, kann es gesamtgesellschaftlich nicht mehr sein, wenn alle Leistungsanbieter dieselbe Position vertreten. Dies würde grosse Probleme für die Patientinnen mit sich bringen, die in der Nähe ihres Wohnorts gesetzlich zulässige Leistungen in Anspruch nehmen (im Rahmen der seit 2002 geltenden neuen Fristenregelung in bezug auf den Schwangerschaftsabbruch). Es darf nicht sein, dass dieser Zugang verhindert oder sehr erschwert wird. Hier handelt es sich um eine Frage der Zivilethik, der Gleichheit vor dem Gesetz und des Zugangs zu ärztlichen Leistungen. In einem solchen Fall ist die Verantwortung der öffentlichen Hand, dabei insbesondere die der gesundheitspolitischen Behörden (Regierungsrat, Kantonsarzt) gefragt. Diese Stellen müssen auf die eine oder andere Weise dafür Sorge tragen, dass den Frauen der Region das entsprechende Leistungsangebot zur Verfügung steht.

Eine jüngste Motion im Grossen Rat des Kantons Waadt fordert die gesetzliche Garantie des Rechts auf Leistungsverweigerung. In einigen
Bereichen ist der Erlass von Gesetzen über die letzten zwanzig Jahre als Fortschritt zu werten beispielsweise was die Beziehung Patient/Betreuer oder die Rechte des Patienten anbelangt. Die Standesregeln haben jedoch weiterhin ihre Bedeutung, und sie anerkennen das Recht auf Verweigerung aus Gewissensgründen. Daher stellt sich die Frage, ob ein solches Gesetz tatsächlich erforderlich ist, zumal das konkrete Handeln der medizinischen Betreuer durch Gesetze nicht umfassend reglementiert wird. In der Tat können im Bereich der medizinischen Betreuung ins Detail gehende Vorschriften zur Belastung werden. Die Notwendigkeit einer solchen Gesetzgebung ist m. E. nicht nachgewiesen. Ausserdem müsste sie den vorbeschriebenen Ausnahmefällen Rechnung tragen, so dass sie zwangsweise kompliziert und ihre Wirkung umstritten wäre - all dies zur Regelung einer Sachlage, die in der Praxis heute keinerlei Probleme bereitet (in meiner offiziellen Amtszeit kam es zu keinem diesbezüglichen Streitfall).

Es stellen sich auch Fragen zur institutionellen und arbeitsrechtlichen Verantwortung. Wie reagieren die Verantwortlichen eines Spitals, wenn sich beispielsweise ein Grossteil des Pflegepersonals weigern würde, bestimmte Handlungen zu vollziehen? Wenn ein oder zwei Mitarbeitende von zwanzig oder dreissig in Gewissensnöten ist bzw. sind, kann dies im Dialog gelöst werden. Was aber, wenn dies bei zehn oder fünfzehn der Fall ist. Welche Position soll dann Vorrang haben? Zumindest muss die Institution ihrer Pflicht in bezug auf den Dienst am Patienten gerecht werden.

Ich unterstütze das deontologische Prinzip der Leistungsverweigerung aus Gewissensgründen. Der vorgeschlagene parlamentarische Weg macht jedoch deutlich, welche Überlegungen es noch anzustellen gilt, bevor nach dem Staat gerufen wird, um in Berufsgattungen oder ins Leben der Bürger einzugreifen. Fazit: Respektierung des ärztlichen Gewissens, ohne Zweifel. Gleichzeitig muss aber auch bedacht werden, dass die Verantwortung, die der Arzt gegenüber seiner Institution und gegenüber der Bevölkerung übernommen hat, dem Recht auf Leistungsverweigerung legitime Grenzen setzen kann. Dabei versteht sich von selbst, dass der Arzt keine beruflichen Verpflichtungen eingehen sollte, bei denen er in solche Gewissensnöte kommt.

Jean Martin * mitglied, ist ehemaliger Kantonsarzt des Kantons Waadt und Mitglied der Nationalen Ethikkommission. 